\title{
Advances in 193 nm Lasers for Fiber Bragg Grating Manufacturing
}

\author{
Ralph Delmdahl ${ }^{1}$, Hans-Gerd Esser ${ }^{1}$, Burkhard Fechner ${ }^{1}$, Remco Nieuwland ${ }^{2}$, Per Karlsson ${ }^{3}$ and Robert Rylander ${ }^{3}$ \\ ${ }^{1}$ Coherent LaserSystems GmbH \& Co. KG, Hans-Boeckler-Str. 12, 37079 Goettingen, Germany \\ ${ }^{2}$ Hittech Multin BV, Laan van Ypenburg 60, 2497 GB Den Haag, The Netherlands \\ ${ }^{3}$ NorthLab Photonics AB, Vattenverksvägen 8, 13141 Nacka, Sweden \\ E-mail: ralph.delmdahl@coherent.com
}

\begin{abstract}
Ongoing progress in the manufacturing of fiber Bragg gratings is to a large extent driven by ultra-short wavelength excimer lasers at $193 \mathrm{~nm}$. This paper will introduce the latest improvements achieved in the development of compact high repetition rate excimer lasers and elaborate on the impact FBG writing instrumentation. Performance and lifetime measurements obtained in a long-term endurance test over the course of 18 months will be shown and discussed. In order to open up the path from laboratory space to broad market industrial use, sufficient component lifetimes and longterm stable performance behavior of the excimer laser have to be ensured. The results shown are based on diverse $193 \mathrm{~nm}$ excimer laser tube improvements aiming at optimizing the gas flow dynamics. This has led to extending the operational life of such compact $193 \mathrm{~nm}$ excimer laser tubes for the first time over several billion pulses even under high duty-cycle conditions
\end{abstract}

DOI: $10.2961 /$ jlmn.2016.03.0021

Keywords: excimer laser, fiber Bragg grating writing, 193 nm, optical sensors, phase mask

\section{Introduction}

Fiber Bragg grating (FBG) technology has been in use since many decades. Commoditization of FBGs, however, has not been fully realized and price tags for FBG devices are still rather high. The reason is that fabrication of FBGs occurs mostly on a small scale and with high unit costs due to the fragmented FBG markets.

Recent improvements concerning the FBG writing system i.e. flexible automation of fiber and mask handling design address these aspects and drive FBG manufacturing which are nowadays mostly employed in optical sensing applications toward widespread commercial use. Using the $193 \mathrm{~nm}$ wavelength in FBG writing has been shown to deliver high material coupling and thus facilitates permanent refractive index change for medium pulse energy levels [1].

The high pulse energy supports sufficient laser fluence levels for permanent refractive index change in optical fibers. Pulsed excimer lasers are the most powerful $193 \mathrm{~nm}$ laser sources on the market and have a history of success in the most demanding industrial applications such as photolithography, refractive eye surgery and high quality laser engraving of transparent materials [2]. Pulse energies of some 5 to several $100 \mathrm{~mJ}$ are available from excimer lasers for automated FBG writing systems.

An FBG is fabricated by producing a periodic modulation in the refractive index of the core of an optical fiber. The resulting periodic pattern creates a Bragg grating that acts as a filter reflecting the incident optical field because of interference [3]. FBG properties such as magnitude, center wavelength and spectral bandwidth of the reflectance are controlled by varying the grating parameters. Specifically, these include the grating period, depth of refractive index modulation and FBG length. Latest technical advances in compact excimer laser development for industrial grade FBG writing systems will be discussed.

\section{Laser design advances}

The latest $193 \mathrm{~nm}$ excimer laser design advances and features pertaining to the generation of the gas discharge conditions and thus the optical output performance will be discussed in the following subsections.

\subsection{Corona preionization}

The preionization concept of compact $193 \mathrm{~nm}$ excimer laser discharge units has been optimized in order to obtain high pulse energy in combination with homogeneous discharge conditions. A new corona preionization design uniquely combines the efficiency of a discharge driven but locally inhomogeneous spark preionization source with the much better smoothness and ionization homogeneity as solely provided by a seamless corona type preionization.

\subsection{Solid state pulsed circuit}

To provide the high pump energy densities in a short time interval as required for population inversion, $193 \mathrm{~nm}$ excimer lasers are discharge pumped using a high-voltage capacitor circuit (pulser) that discharges the stored electrical energy directly into the active ArF medium. The pumping scheme involves efficient switching of this stored electrical energy into the discharge system in a very short time. To obtain the required peak current and voltage rise time, excimer lasers use multi-stage pulse compression and allsolid-state switching through modern semiconductor switches such as IGBTs (insulated gate bipolar transistor).

The primary solid state switching circuit transfers the energy on a slow time scale from the primary energy store (C0) to intermediate energy stores in a multiple stage magnetic compression circuit, from where it is then rapidly transferred into the laser cavity for the discharge. In compact excimer lasers for FBG writing, all-solid-state switch- 
es constitute a major advancement towards highly reliable and cost-efficient industrial excimer laser systems as they are maintenance-free and have demonstrated a practically unlimited lifetime.

\subsection{Laser tube and gas discharge design}

A transverse circulation fan positioned within the laser tube replaces the gas volume between the main electrodes completely after each laser pulse, thereby providing a homogeneous gas flow over the entire electrode length. Between consecutive laser pulses the gas exchange in the discharge must provide clearing by at least a factor of two. Excimer lasers typically operate with some $4 \%$ conversion efficiency between the electrical input power and the UV output power; the surplus energy is removed efficiently as excess heat.

Air-cooling up to $500 \mathrm{~Hz}$ pulse repetition rate is achieved in compact $193 \mathrm{~nm}$ excimer lasers designed for integration in FBG writing instruments. Depending on the duty-cycle of use higher pulse frequencies are realized with additional water-cooling of the laser discharge unit. The forced circulation in the laser tube brings the laser gas, heated up by the laser discharge, to a heat exchanger always providing the correct operating temperature of the active medium. The laser tube windows, for optimum output, must be protected against contamination from electrochemical erosion processes in the discharge.

\section{Results}

The technical advances in the laser design have led to performance advances which will be introduced and discussed in the following sections.

\subsection{Component lifetimes}

Laser tube and window lifetimes of up to 7 billion consecutive laser pulses were achieved in endurance testing employing a high voltage stabilized pulse energy of $5 \mathrm{~mJ}$ at a wavelength of $193 \mathrm{~nm}$ and a pulse frequency of $1000 \mathrm{~Hz}$. As soon as the high voltage achieved about $85 \%$ of its maximum, a gas exchange was performed leading to a drop in the high voltage necessary to stabilize the laser at $5 \mathrm{~mJ}$ pulse energy. The respective high voltage curve recorded during the $5 \mathrm{~mJ}$ stabilized energy run is shown in figure 1 below. A single set of resonator windows was used in the endurance measurement. A new gas fill was performed at each maximum of the high voltage curve. Arrows indicate regular optics cleaning intervals.

In order to extend the hands-off operation time of compact excimer lasers in view of automated mass spectrometry related applications, the interior architecture of the gas reservoir including gas flow architecture and the internal electrostatic gas purification system have been redesigned. Laser gas contaminants are trapped efficiently by careful optimization of the gas flow via capable electrostatic filter elements. As a result, gas performance remains unaffected during long-term, hands-free, stabilized energy operation over the course of many millions of laser pulses.

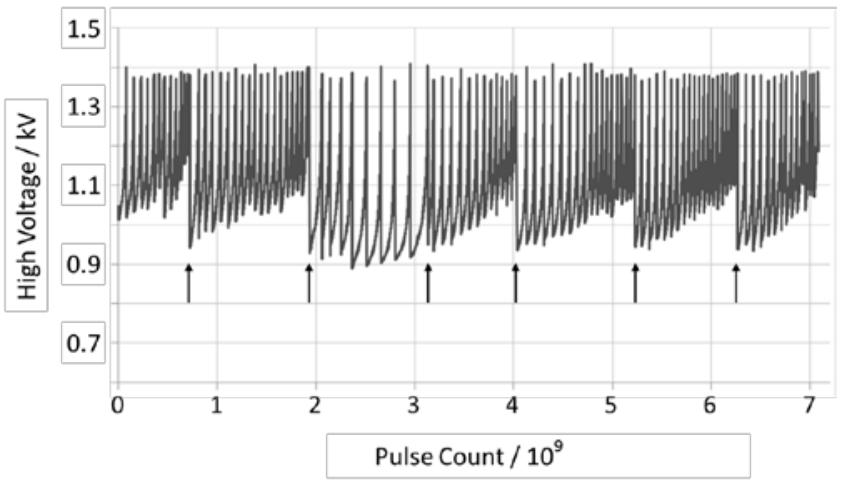

Fig. 1 Endurance test over more than 7 billion consecutive laser pulses stabilized at $5 \mathrm{~mJ} /$ pulse output energy at $193 \mathrm{~nm}$.

\subsection{New fill intervals}

Figure 2 shows a measurement of the laser discharge voltage behavior at a stabilized pulse energy of $5 \mathrm{~mJ}$ and a repetition rate of $1000 \mathrm{~Hz}$. The stabilized energy run was started with fresh laser gas and could be extended over more than 14 million laser pulses with the same gas fill.

The laser gas consumed for the gas lifetime of 14 million laser pulses amounts to only 16 liters. Moreover, a factor of two reduction of gas consumption is achieved by means of consecutive on-the-fly premix gas injections during laser operation. Gas aging effects are compensated via discharge voltage increase which makes it necessary to exchange the laser gas at a preset upper voltage threshold after in this case 15 million shots.

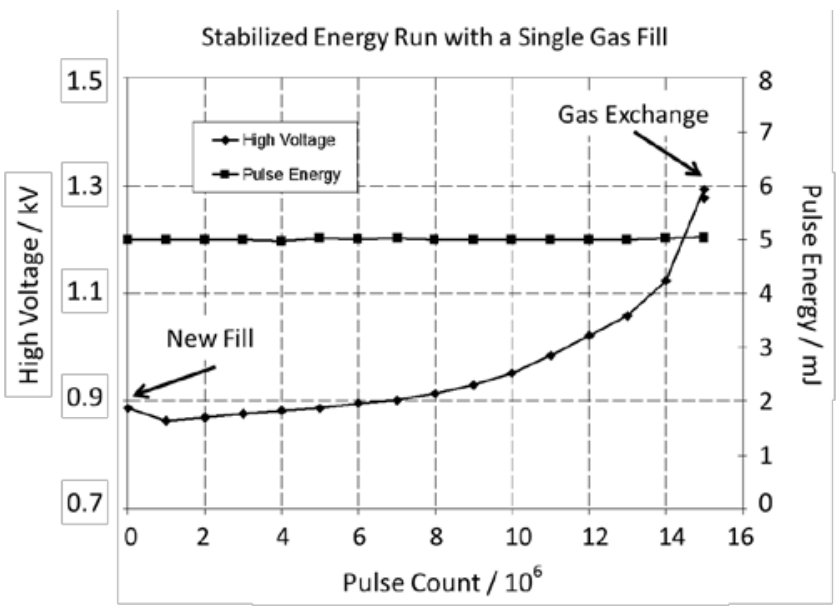

Fig. 2 Laser discharge voltage behavior during a gas life run at $5 \mathrm{~mJ}$ stabilized and $1000 \mathrm{~Hz}$ pulse frequency at $193 \mathrm{~nm}$.

The pulse energy stability of the $193 \mathrm{~nm}$ excimer laser during $500 \mathrm{~Hz}$ operation at typical high voltage levels between 1.5 and $0.9 \mathrm{kV}$ is always better than $1 \%$, rms. As visible from the measurement shown in figure 3 , the energy fluctuations start to rise below high voltage levels of $9 \mathrm{kV}$. This effect is due to the laser resonator approaching the lasing threshold when operated near the minimum of the selectable high voltage range. 


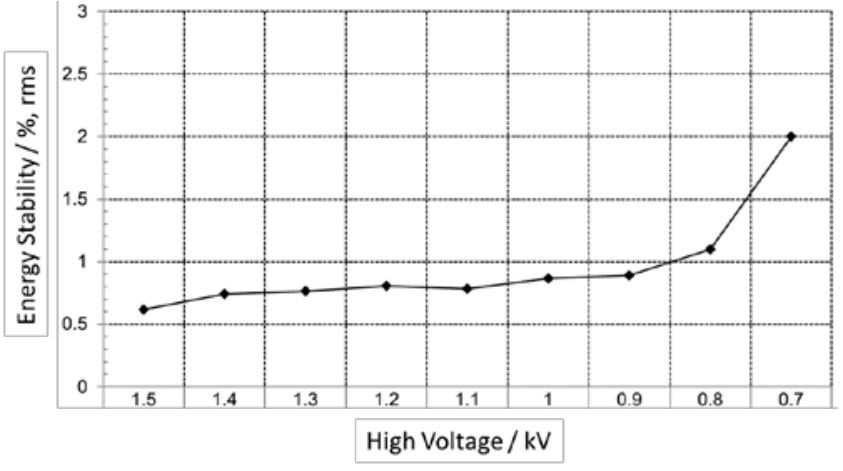

Fig. 3 Pulse energy stability versus high voltage at $500 \mathrm{~Hz}$.

\section{Flexible automation in FBG writing}

Next to $193 \mathrm{~nm}$ excimer laser performance and lifetime advances, the architectural design of writing systems for FBGs must be capable of reducing the unit costs in FBG production and delivering FBGs with excellent unit-to-unit consistency thus, simplifying the task of flexible, high volume production for the emerging and highly fragmented optical sensor market. A system approach balancing volume production and flexibility in grating parameters for phase mask FBG writing is discussed in the next sections.

\subsection{Systems for flexible phase mask writing of FBGs}

FBG fabrication usually occurs via the phase mask method exposing a photosensitive fiber to an interference fringe pattern created by a phase mask. Directing the excimer laser beam through a phase mask the latter diffracts the incident laser light into various orders, which overlap and optically interfere with each other in a region of some $100 \mu \mathrm{m}$ behind the mask. This results in alternating areas of laser intensity along the fiber core, whose spacing is either equal to the phase mask period, or half of this value [4], depending upon the exposure geometry (Fig. 3).

While this method is conceptually straightforward, in the real world, there are several significant barriers to overcome when producing FBGs.

The first is holding and positioning all the components such that a grating having precisely the right spacing and index variation characteristics is generated at exactly the correct place along the fiber. Furthermore, if the goal is to produce large amounts of FBGs, each having consistent characteristics, a FBG writing system based on $193 \mathrm{~nm}$ excimer technology must be capable of accommodating batch-to-batch variations in the index of the optical fiber used. Automated systems must provide cost effective, flexible manufacturing of high quality, consistent FBGs.

A recent system approach integrating an excimer laser operating at $193 \mathrm{~nm}$, beam conditioning optics, up to 16 phase masks (uniform or chirped), automated mechanics and control software for flexible FBG mass manufacture is shown in figure 4.
The optical fiber is led through a fiber fixture allowing FBGs to be written at precise positions.

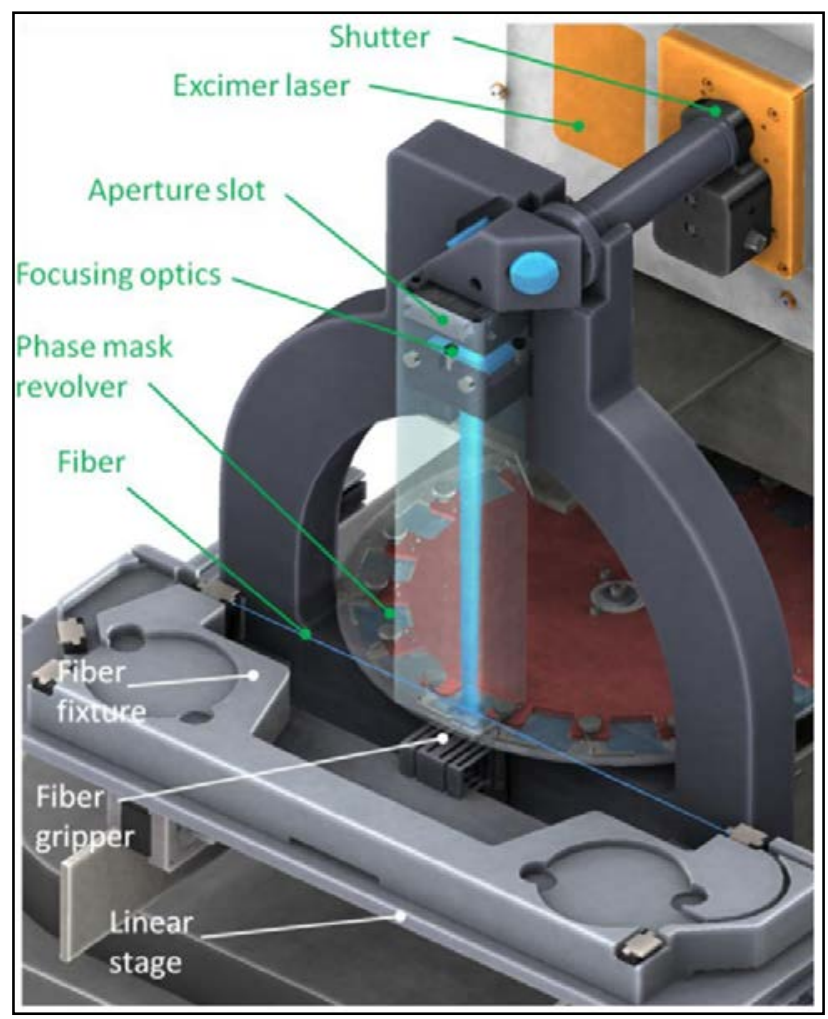

Fig. 4 Flexible automation of phase mask FBG writing

The refractive index change in the case of germanosilicate fibers (SM1500 series from Fibercore) having three different Ge dopant levels is shown in figure 5. A few thousand excimer laser pulses are typically applied, in order to achieve sufficient grating reflectivity with the number of pulses depending on the dopant level.

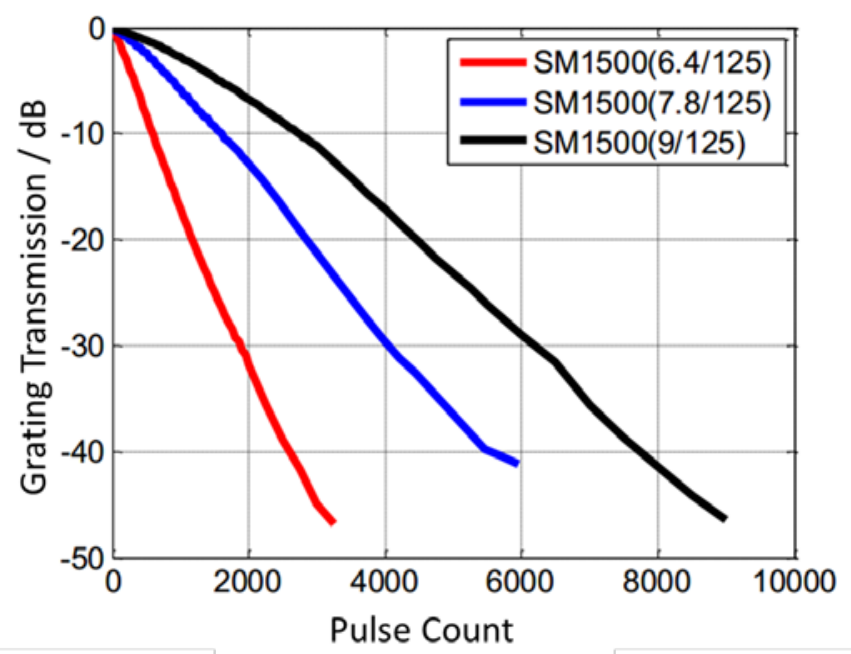

Fig. 5 Grating transmission as a function of pulse number.

\subsection{Accuracy of the FBG center wavelength}

Among the most critical parameter for an FBG is the center wavelength. This quantity is determined by two parameters - the phase mask and the effective refractive index of the fiber. 
The effective refractive index is derived from the fiber's numerical aperture (NA), the value of which is specified by the manufacturer for every fiber. As manufacturers do not tightly control the core refractive index, result is significant variations for different fiber lots.

Reproducible center wavelength values despite variations in the refractive index of a $125 \mu \mathrm{m}$ fiber are achieved by applying a force to physically stretch the fiber during the writing process using the fiber fixture (Fig 6). The pretension force thus accounts for NA variations.

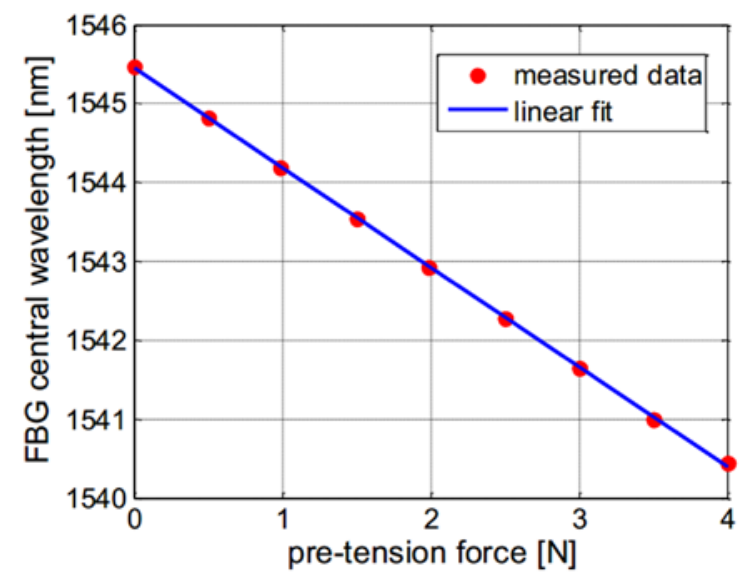

Fig. 6 Grating transmission as a function of pulse number.

In practice, shifting FBG center wavelength as much as $4 \mathrm{~nm}$ for a phase mask nominally centered at $1550 \mathrm{~nm}$ is usually achieved, which is more than twice what is necessary to correct for the typical batch-to-batch variations in fiber numerical aperture. Control of the pre-tension force is precise enough to deliver center wavelength accuracy of $100 \mathrm{pm}$, and repeatability of $\pm 50 \mathrm{pm}$ in production.

\subsection{Reflectance bandwidth}

For a grating with a uniform index modulation spectral bandwidth narrows with increasing grating length as shown in figure 7. However, the sharper the reflectance peak, the more energy is shunted into the side lobes. This effect can be mitigated by varying the depth of index modulation along the FBG, which is called apodization. However, this does produce some increase in center reflectance peak bandwidth because it decreases the effective grating length.

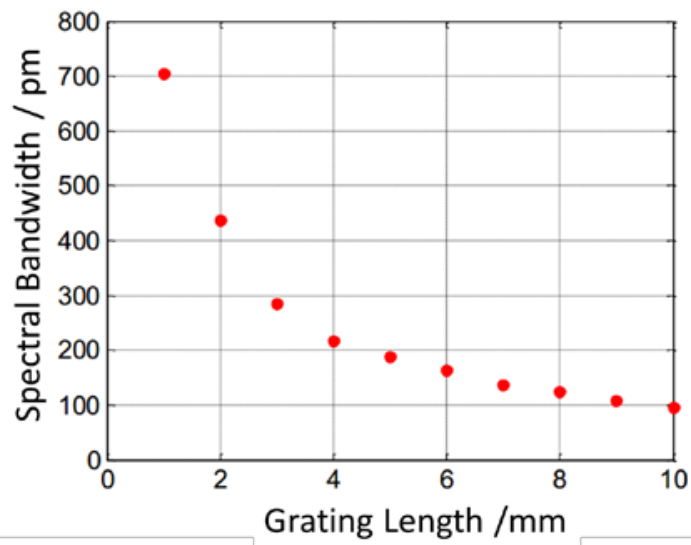

Fig. 7 Effect of grating apodization for side lobe supression.
The $193 \mathrm{~nm}$ excimer laser has a uniform (top hat) intensity distribution in the long direction, and a near-Gaussian profile in the short dimension. High beam coherence optics for the excimer laser beam will shape the excimer laser beam such that the system can write FBGs from typically 1 to 10 $\mathrm{mm}$ in length. From a technical viewpoint, the capability for beam apodization is mandatory and is accomplished by using a shaped aperture in the beam path to convert the uniform distribution of the laser into a Gaussian profile. For example, a Gaussian apodization mask can achieve a side lobe suppression ratio of typically $15 \mathrm{~dB}$ for a $10 \mathrm{~mm}$ length FBG.

\subsection{Mechanical stability and positional accuracy}

Writing a uniform FBG with a center wavelength transmission reduction of $20-50 \mathrm{~dB}$ requires as many as 1000 laser pulses at an energy of $5 \mathrm{~mJ} /$ pulse from the 193 $\mathrm{nm}$ excimer laser. At a repetition rate of $500 \mathrm{~Hz}$, this would imply an exposure time of just two seconds. Chirped FBGs where the grating period changes along the length of the grating require much higher refractive index contrast to reach the same level of reflectance, meaning even longer exposure times. Any relative motion between the phase mask and fiber over this period, even at the nanometer level, is enough to degrade FBG performance. In order to eliminate any relative vibrational motion in a FBG writing system, the fiber must be positioned relative to phase mask with submicron accuracy during exposure. However, the fiber holding and clamping system must meet another important requirement. For sensing applications, in particular, usually multiple FBGs are written into a single fiber [5]. The spacing between these individual FBGs usually have to be controlled very precisely. Fiber positioning technology is thus as important in the case of multiple gratings to be inscribed. Translation stage positional accuracy enabling multiple FBGs to be written over a total fiber length of $250 \mathrm{~mm}$ has been achieved recently to be of the order of $0.1 \mathrm{~mm}$.

\section{Conclusion}

In conclusion, widespread deployment of FBGs has been limited up to now partly by the expense and difficulty of device fabrication. Excimer laser performance advances in conjunction with new system approaches for turnkey, fast and flexible FBG writing with unprecedented accuracy and reproducibility will open up new opportunities in the emerging optical sensing markets.

\section{References}

[1] Y. Ran, Y. N. Tan, L. P. Sun, S. Gao, J. Li, L. Jin, and B. O. Guan: Opt. Exp., 19, (2011) 18577.

[2] R. Delmdahl: Nature Photonics, 4, (2010) 286.

[3] K. O. Hill, B. Malo, F. Bilodeau, D. C. Johnson, and J. Albert: Appl. Phys. Lett., 62, (1993) 1035.

[4] Y. Wang, J. Grant, A. Sharma, and D. Myers: J. Lightwave Technol., 19, (2001) 1569.

[5] R. Di Sante, L. Donati, E. Trojani, and P. Proli: Met. Mater. Int., 20, (2014) 537.

(Received: May 9, 2016, Accepted: August 9, 2016) 